\title{
Criminologie
}

\section{Fonctionnement sectaire et violence envers les enfants : le cas de l'Église baptiste de Windsor}

\section{Adriana Pacheco et Dianne Casoni}

Volume 41, numéro 2, automne-hiver 2008

Les organisations dites sectes, les lois et la société

URI : https://id.erudit.org/iderudit/019433ar

DOI : https://doi.org/10.7202/019433ar

Aller au sommaire du numéro

\section{Éditeur(s)}

Les Presses de l'Université de Montréal

ISSN

0316-0041 (imprimé)

1492-1367 (numérique)

Découvrir la revue

Citer cet article

Pacheco, A. \& Casoni, D. (2008). Fonctionnement sectaire et violence envers les enfants : le cas de l'Église baptiste de Windsor. Criminologie, 41(2), 53-90.

https://doi.org/10.7202/019433ar
Résumé de l'article

Cet article vise, par une analyse de cas unique, à étudier l'évolution d'un groupe religieux minoritaire du Québec, l'Église baptiste de Windsor, vers un fonctionnement sectaire particulier qui l'isola de son environnement social et donna lieu à une grave situation de violence contre des mineurs. Les enfants du groupe y ont été soumis à de sévères punitions corporelles qui furent jugées comme constituant des sévices physiques par les services de protection de l'enfance et par le bureau du procureur général. Grâce à une analyse documentaire exhaustive à laquelle se sont ajoutés des témoignages d'anciens membres du groupe ainsi que des entretiens avec des informateurs clés, la reconstruction de l'histoire du groupe, de sa fondation à sa dissolution, a été rendue possible. Quatre moments dans le développement du groupe ont été identifiés ; leur analyse a permis de cerner les éléments qui ont contribué à la mise en place d'un contexte groupal et doctrinal qui légitimait le recours généralisé aux sévices physiques des enfants y vivant. 


\title{
Fonctionnement sectaire et violence envers les enfants: le cas de l'Église baptiste de Windsor
}

\author{
Adriana Pacheco \\ Doctorante \\ École de criminologie, Université de Montréal \\ adriana.pacheco.espino.barros@umontreal.ca \\ Dianne Casoni \\ Professeure \\ École de criminologie, Université de Montréal \\ dianne.casoni@umontrel.ca
}

RÉSUMÉ - Cet article vise, par une analyse de cas unique, à étudier l'évolution d'un groupe religieux minoritaire du Québec, l'Église baptiste de Windsor, vers un fonctionnement sectaire particulier qui l'isola de son environnement social et donna lieu à une grave situation de violence contre des mineurs. Les enfants du groupe y ont été soumis à de sévères punitions corporelles qui furent jugées comme constituant des sévices physiques par les services de protection de l'enfance et par le bureau du procureur général. Grâce à une analyse documentaire exhaustive à laquelle se sont ajoutés des témoignages d'anciens membres du groupe ainsi que des entretiens avec des informateurs clés, la reconstruction de l'histoire du groupe, de sa fondation à sa dissolution, a été rendue possible. Quatre moments dans le développement du groupe ont été identifiés; leur analyse a permis de cerner les éléments qui ont contribué à la mise en place d'un contexte groupal et doctrinal qui légitimait le recours généralisé aux sévices physiques des enfants y vivant.

\section{Introduction}

Cet article vise, par une analyse de cas unique, à étudier l'évolution d'un groupe religieux minoritaire du Québec, l'Église baptiste de Windsor, vers un fonctionnement sectaire particulier qui l'isola de son environnement social et favorisa un passage à l'acte violent envers les enfants du groupe. Ceux-ci y ont été soumis à de sévères punitions 
corporelles qui furent jugées comme constituant des sévices physiques par les services de protection de l'enfance et par le bureau du procureur général de la province.

L'intérêt de cette recherche provient d'abord et avant tout du fait que l'évolution du groupe, et de la dérive qu'il a connue, ont pu être étudiées depuis sa fondation jusqu'à sa dissolution. Il est en effet rare d'accéder à suffisamment de documents et d'acteurs clés pour pouvoir reconstruire l'histoire de groupes qui connaissent une dérive violente. Il y a lieu de rappeler que les connaissances sur les dérives sectaires proviennent en grande partie, sinon presque exclusivement, d'études de cas, en raison de la rareté relative de tels phénomènes, particulièrement au Canada. Par exemple, c'est grâce à une étude de cinq cas que Bromley (2002) a pu contribuer de manière significative aux connaissances sur les dérives sectaires, qu'il a appelées «dénouements tragiques». Ces connaissances sont précieuses compte tenu des enjeux en cause, particulièrement lorsque des enfants sont concernés.

Dans le cas présent, l'étude de l'évolution de l'Église baptiste de Windsor vise à comprendre plus précisément le type de processus qui conduit un leader à imposer une orientation doctrinale favorisant le passage à l'acte violent envers les enfants du groupe. De façon complémentaire, cette étude vise aussi à comprendre comment les parents des enfants impliqués peuvent en venir à accepter pareils traitements infligés à leurs enfants. Dans un contexte où le savoir sur ces questions est limité, tout apport nouveau est utile car susceptible de contribuer à éviter de tels passages à l'acte violents à l'encontre d'enfants.

Avant de présenter le cadre théorique choisi pour l'analyse de ce cas, une courte section précisera la terminologie utilisée. Puis les modèles théoriques retenus comme étant les plus pertinents pour contextualiser la reconstruction historique établie seront présentés. Une section consacrée à la description de la méthodologie suivra. Ensuite, une reconstruction historique partielle du groupe, destinée à décrire son évolution vers une situation de graves sévices envers les enfants, sera présentée. Enfin une discussion et une conclusion suivront.

\section{Précisions terminologiques}

Avant de poursuivre, il est important de préciser le sens attribué à plusieurs notions concernant l'orientation doctrinale plus large dans 
laquelle l'Église à l'étude s'inscrit, non seulement afin d'éviter tout malentendu, mais aussi afin que le lecteur dispose des précisions terminologiques nécessaires pour bien comprendre tant la reconstruction historique présentée que son analyse.

Deux orientations doctrinales principales sont distinguées au sein du protestantisme. La première se définit comme étant libérale et la seconde se désigne comme étant conservatrice. Cette dernière, qui caractérise l'Église baptiste de Windsor, se traduit par sa fidélité à des préceptes conçus comme étant fondateurs du protestantisme, en particulier la croyance que la Bible est la parole directe de Dieu et que, de ce fait, elle est infaillible et constitue la source fondamentale de toute autorité. Il s'ensuit que la Bible est vue non seulement comme devant être mise en application de manière stricte, mais encore comme devant régir la conduite des êtres humains dans toutes les sphères de leur vie. Le protestantisme conservateur n'est pas associé à une dénomination confessionnelle précise; toutefois les Églises baptistes, évangéliques et pentecôtistes en font généralement partie, mais pas nécessairement (Bergeron, 1987; Ellison et Sherkat, 1993 ; Chartrand, 1995). Le degré de conservatisme des individus et des groupes varie en fonction du type d'interprétation fait de la Bible; plus cette interprétation se rapproche du texte, plus elle est associée au fondamentalisme.

Le fondamentalisme représente la position la plus conservatrice au sein du protestantisme conservateur. Apparu à la fin du XIX $x^{e}$ siècle aux États-Unis à la suite de conflits entre protestants conservateurs et libéraux liés, entre autres, au positionnement des Églises protestantes par rapport à la question des théories évolutionnistes, le fondamentalisme se caractérisa, dès sa naissance politique, par son radicalisme en matière de doctrine et par son parti pris de fonder l'ordre social sur les préceptes de la Bible. Sur le plan doctrinal, ce courant transconfessionnel s'articule autour d'une vingtaine de principes considérés comme les «fondements» de la foi, dont les plus importants sont la croyance en l'inerrance absolue de la Bible et le biblisme, c'est-à-dire l'interprétation - et en conséquence l'application - littérale de la Bible. À cela s'ajoutent l'importance de l'expérience de la conversion, le devoir de s'engager dans un prosélytisme vigoureux et la présence d'un fort rigorisme moral qui vise à restaurer la vertu dans la société. En outre, une eschatologie millénariste catastrophiste qui vise à ramener le Royaume de Dieu sur terre et la conviction d'avoir un rôle messianique à jouer dans la bataille finale qui opposera les forces du bien et du mal caractérisent le fonda- 
mentalisme (Bergeron, 1987; Hervieu-Léger, 1988; Ben Barka, 1998; Chartrand, 1995; Woodberry et Smith, 1998; Fath, 2004).

\section{Contextualisation théorique}

La contextualisation théorique qui encadre cette recherche est divisée en trois sections distinctes. Une première présentera brièvement le concept d'univers symbolique de Berger et Luckman (1966) et servira à mieux comprendre la place du discours du protestantisme conservateur dans les groupes religieux qui se réclament de cette orientation. Une recension des principaux écrits au sujet de la position du protestantisme conservateur sur la punition corporelle constituera la seconde section de cette contextualisation et sera complétée par une courte présentation de recherches sur les effets de ce discours sur cette pratique. Enfin, la troisième section présentera un modèle théorique décrivant deux types de fonctionnement groupal observés dans des groupes religieux minoritaires qui sont susceptibles de connaître une dérive violente.

\section{Le concept d'univers symbolique}

Comme le rappellent Berger et Luckmann (1966), l'institutionnalisation des pratiques au sein des groupes est possible grâce à un discours qui les légitime. Parmi les degrés de légitimation qu'ils proposent figure la légitimation en vertu d'un univers symbolique, telle la religion, qui est celui qui assure le mieux le maintien des pratiques dans un groupe social. La légitimation par un univers symbolique offre à ces pratiques une justification en faisant appel à un ordre qui transcende la vie quotidienne (dans le cas de la religion, à un ordre surnaturel) en leur attribuant un sens qui dépasse celui de leur utilité pragmatique. Même si la pratique en question est inefficace, elle sera maintenue grâce à la croyance des individus dans l'univers symbolique qui la justifie, précisent Berger et Luckman. À leur tour, certaines pratiques contribuent à la conservation de l'univers symbolique, et, de ce fait, sont privilégiées. Ainsi, le sens donné à une pratique tendra à être positif dans le cas de celles qui servent à renforcer l'univers symbolique d'un groupe, ce qui favorisera son institutionnalisation éventuelle, alors qu'il sera négatif dans le cas où cette pratique l'affaiblirait (Berger et Luckmann, 1966). 


\section{Discours du protestantisme conservateur sur la punition corporelle ${ }^{1}$}

Le discours contemporain du protestantisme conservateur au sujet de la punition corporelle s'appuie sur une représentation négative de l'enfant héritée du calvinisme (Illick, 1974; Waltzer, 1974). Invoquant la tache du péché originel, l'enfant est vu comme ayant une nature corrompue et encline au mal qui le prédispose à l'égoösme et à la rébellion à l'égard de toute forme d'autorité. En revanche, comme l'enfant est aussi vu comme une créature de Dieu, la tâche première des parents est de le sauver du mal qui l'habite. Ainsi, une guerre permanente entre les forces attribuées au bien et celles attribuées au mal est conçue comme se déroulant dans l'âme de l'enfant. Dobson (1976, 1987), l'un des penseurs protestants conservateurs qui participe le plus activement à l'institutionnalisation de la pratique de la punition corporelle aux États-Unis souligne que les parents doivent s'attaquer à l'esprit rebelle de l'enfant dès son plus jeune âge; il les exhorte à «frapper le cœur de l'enfant» de manière énergique afin d'y chasser l'obstination et de renverser sa propension au péché. Le meilleur moyen d'y parvenir, selon Dobson et d'autres théoriciens du protestantisme conservateur comme Jerry Falwell ou Beverly et Tim LaHaye serait de pratiquer une «discipline diligente» à l'aide du châtiment corporel administré avec un instrument. À cet effet, la verge est identifiée comme la première réponse de Dieu - telle qu'elle est transmise dans la Bible - pour contrer la rébellion enfantine selon le discours protestant conservateur (Dobson, 1976, 1987; Falwell, 1981 ; LaHaye, B., 1977; LaHaye, T., 1980).

Les Protestants conservateurs appuient leur pratique du châtiment corporel sur quelques versets bibliques interprétés comme une prescription divine, notamment les versets $22: 15$ et $23: 13-14$ :

La folie est liée au cœur de l'enfant.

La verge de la correction l'éloigne de lui.

(La Sainte Bible, verset 22:15)

1. L'expression punition corporelle sera utilisée indistinctement de celle de châtiment corporel puisqu'elles décrivent exactement l'acte dont il est question. Toutes les deux rendent compte du caractère punitif de l'acte et du fait qu'il comporte une atteinte souhaitée au corps de l'enfant dans le but de lui infliger une douleur. Les penseurs du protestantisme conservateur, pour leur part, préfèrent le mot discipline (en anglais corporal discipline) pour distinguer leurs pratiques de celles qui ne sont pas inspirées d'une justification biblique. Ils considèrent que les punitions corporelles qui ne sont pas inspirées de la Bible peuvent être dommageables pour l'enfant en raison des excès auxquels elles peuvent mener (Despatie, 2005). 
N'épargne pas la correction de l'enfant;

Si tu le frappes de la verge, il ne mourra point.

En le frappant, tu délivres son âme du séjour des morts.

(La Sainte Bible, verset $23: 13-14$ )

L'enfant doit être contraint à l'obéissance, rappelle-t-on aux parents en chaire, afin qu'il accepte de se soumettre d'abord à l'autorité parentale puis, plus tard, à la volonté de Dieu. La punition corporelle est d'ailleurs présentée dans le discours protestant conservateur comme le meilleur moyen de chasser l'influence de Satan du cœur des enfants. De surcroît, le recours au châtiment corporel est considéré comme une preuve de l'amour parental, comme la référence au prochain verset fréquemment rappelé dans le discours des Protestants conservateurs l'illustre bien:

Celui qui épargne la verge haït son fils.

Mais celui qui l'aime met de la diligence à le discipliner.

(La Sainte Bible, Proverbe $13: 24$ )

La portée du discours des ténors protestants conservateurs auprès des parents ne fait pas de doute d'après les résultats des recherches à ce sujet $^{2}$. En effet, l'acceptation de la punition corporelle des enfants est plus grande chez les parents appartenant à des groupes protestants conservateurs que chez ceux qui appartiennent à d'autres groupes religieux ou qui n'appartiennent à aucun groupe religieux (Erlanguer, 1974; Grasmick et al., 1991, 1992 ; Ellison et Sherkat, 1993 ; Bartkowski, 1995; Ellisson, 1996; Ellison et al., 1996; Danso et al., 1997; Wilcox, 1998 ; Woodberry et Smith, 1998). Plus précisément, il appert que les Protestants conservateurs ont une attitude significativement plus favorable à la punition corporelle des enfants que les individus appartenant à d'autres orientations religieuses et qu'ils s'en servent plus fréquemment. Ellison et Sherkat (1993) notent, en outre, que plus l'interprétation de la Bible est littérale, plus les parents sont portés à utiliser la punition corporelle comme moyen pour corriger leurs enfants.

Ellison et al. (1996) notent, par ailleurs, que les Protestants conservateurs sont portés à administrer des punitions corporelles plus sévères

2. La majorité de ces recherches ont été réalisées aux États-Unis, où le protestantisme conservateur a vu le jour et où il est plus répandu, mais deux ont été menées au Canada anglophone par Danso et al. (1997). Les résultats de ces recherches sont congruents avec ceux des travaux menés aux États-Unis. À notre connaissance, la seule étude comparable menée au Québec est celle entreprise par Casoni auprès d'un large échantillon d'étudiants universitaires dont les principaux résultats peuvent être consultés pour le moment dans Despatie (2005). Ce texte offre aussi une recension plus complète des écrits sur le sujet. 
que les autres parents, notamment parce qu'ils utilisent des instruments pour frapper les enfants. De plus, ils auraient tendance à interpréter une foule de comportements - habituellement jugés normaux par les parents, compte tenu du degré de développement de l'enfant - comme des manifestations dites de rébellion enfantine, donc punissables par châtiment corporel. Ils auraient tendance à interpréter des manifestations comme des pleurs engendrés par la fatigue, des accès d'activité motrice ou encore des symptômes de maladie qui rendent l'enfant irritable, pleurnichard ou somnolent comme autant d'expressions de l'esprit de rébellion contre lequel on les met en garde. Cette tendance à interpréter des comportements courants comme de la rébellion favoriserait un recours fréquent au châtiment corporel qui, à son tour, conduirait à une escalade dans la sévérité des punitions, remarque Ellison (1996). Le recours à des instruments pour administrer les châtiments corporels augmenterait considérablement le risque de lésions, soulignent de surcroît Bartkowski et Ellison (1995) à la lumière de leurs données. Dans ce sens, il appert que le discours sur le châtiment corporel des Protestants conservateurs participe très certainement à renforcer l'univers symbolique de certaines congrégations, dont certaines congrégations baptistes comme celle à l'étude. Plus précisément, en ce que la pratique de la punition corporelle y reçoit, depuis fort longtemps déjà, une sanction positive puisqu'elle est perçue comme le moyen privilégié par Dieu pour chasser les forces malignes (evil) de l'âme de l'enfant.

\section{Philosophies groupales}

Quant aux types de fonctionnement adoptés par des groupes religieux minoritaires susceptibles d'être associés à une dérive violente, une théorisation en particulier apparaît pertinente pour l'étude du cas de l'Église baptiste de Windsor. Il s'agit du modèle dit des philosophies groupales proposé par Casoni (2000), qui décrit quatre types de fonctionnement distincts vus comme favorisant la dérive violente. Ce modèle présente pour chacune des quatre philosophies isolées les spécificités caractérisant divers éléments de la vie de groupe, soit le type de leadership qui y sera privilégié, le genre de membership recherché, le traitement des enfants qui y aura cours, la doctrine qui s'y développera, le type de rituels qui seront mis en pratique et les genres d'organisation spatiale et sociale privilégiés. 
Par philosophie, Casoni (2001) entend une façon implicite - et parfois même explicite - de voir la vie, de se représenter sa conduite et d'interpréter la conduite des autres. Dans ce sens, la définition six du mot philosophie dans le Nouveau Petit Robert (1994) rend bien compte du sens qui a été accordé à ce mot: «ensemble des conceptions philosophiques communes à un groupe social». Bien que quatre philosophies distinctes aient été isolées et décrites (Casoni, 2000; Casoni et Brunet, 2005), deux seules seront présentées, car celles-ci uniquement apparaissent pertinentes dans l'analyse des données colligées, soit la philosophie dite de séparation et celle dite de pureté, laissant de côté celles dites de survie et d'indulgence. De plus, afin de limiter la longueur de la contextualisation théorique, seuls les éléments jugés pertinents à l'étude présente seront décrits.

En accord avec la philosophie dite de séparation, une démarcation continuelle est recherchée afin de toujours pouvoir distinguer les membres du groupe des autres. Ces groupes encouragent ainsi leurs membres à respecter une grande orthodoxie. Même lorsque des changements de doctrine sont introduits par les leaders, il est attendu que les membres les acceptent sans en remettre en question leur bien-fondé. Une telle obéissance à l'orthodoxie groupale sert notamment à maintenir la cohésion du groupe. Le leadership est souvent partagé par une petite élite dont les individus agissent comme des agents de contrôle social à l'intérieur du groupe. Il est attendu de tous les membres qu'ils maintiennent la cohésion groupale en filtrant constamment les comportements appartenant au nous des comportements appartenant au eux, et ce, non seulement envers eux-mêmes, mais aussi en ce qui concerne leurs enfants, leur famille, leurs voisins et leurs amis (Casoni, 2000).

Les enfants sont prisés pour le rôle qu'ils peuvent jouer dans la transmission de la foi et de la tradition du groupe. Les comportements typiquement enfantins et l'immaturité sont considérés comme inacceptables, étant vu comme des attitudes et des agissements typiques de l'autre - soit des personnes étrangères au groupe. Dans certains groupes, les enfants fautifs, même légèrement, feront non seulement l'objet d'humiliation publique, mais seront aussi parfois exposés à de fréquentes punitions corporelles. Malheureusement, à cause d'une forte pression sociale envers la conformité, il peut arriver dans les groupes particulièrement stricts que certains enfants soient maltraités. Cependant, selon les normes en vigueur au sein de nombre de ces groupes, le recours à 
la punition corporelle, même excessive, est considéré comme correct et justifié (Ibid.).

Ces groupes acceptent habituellement bien la promiscuité géographique avec des non-membres, vivant souvent dans des cadres urbains, ou dans de petites villes, par exemple. Bien que l'immersion sociale ne soit pas la règle, des contacts minimaux avec les non-membres sont acceptés. Cette promiscuité sociale est tolérée puisque la distinction entre le nous et l'eux est garantie par les nombreux signes manifestes qui caractérisent le groupe. En ce qui concerne l'organisation sociale, elle est habituellement patriarcale. Une discrimination stricte entre les rôles et les responsabilités des hommes et des femmes est souvent la règle. Les femmes sont le plus souvent contraintes à adopter une position servile et restreinte à la famille. Bien qu'une hiérarchie de pouvoir existe habituellement, les luttes politiques sont une réalité omniprésente dans l'organisation sociale. Des conflits entourant les questions de pouvoir peuvent éclater entre les membres influents et mener à des crises majeures, dont des scissions (Ibid.).

Quant à la philosophie dite de pureté, elle est décrite par Casoni (2000) comme étant une exacerbation de la philosophie de séparation. Dans de tels groupes, toute chose, qu'elle soit matérielle ou spirituelle, est jugée selon des critères de pureté et d'impureté. Il est donc attendu que les membres cherchent à atteindre l'absolu à travers chaque acte, chaque pensée, et ce, à chaque instant de leur existence. Les caractéristiques humaines telles l'expression d'émotions, de sexualité et la satisfaction des besoins corporels sont une cible de prédilection, étant considérées d'emblée comme des signes de l'échec à atteindre l'idéal de pureté recherché. Souvent, le leadership est entre les mains d'une seule personne, parfois autoproclamée. La structure du pouvoir étant restreinte à une seule personne, un régime totalitaire est souvent en vigueur. Dans ces cas, l'arbitraire est la norme dans l'exercice du pouvoir, ce qui peut néanmoins être très bien accepté par les membres, surtout lorsque le pouvoir a été attribué de façon consensuelle à un individu jugé comme le plus pur, donc le plus sage. Au-delà de la conformité à une orthodoxie, une certaine uniformité en ce qui concerne l'expression des traits individuels de personnalité est observée; l'individualité est ouvertement découragée puisqu'elle est vue comme un signe de vanité ou d'égoïsme, donc de faiblesse humaine. La forte cohésion sociale à l'intérieur de ces groupes est d'ailleurs liée au renoncement à l'expression des individualités propres des membres. 
Lorsqu'il y a des enfants, ils sont souvent à risque d'être maltraités physiquement, la punition corporelle y étant utilisée de façon excessive puisque l'absolu règne. Une négligence peut aussi être présente sous forme de manque de nourriture, d'eau, de sommeil, de chaleur ou de soins médicaux puisqu'il est attendu des enfants, tout autant que des adultes, qu'ils ignorent la satisfaction de leurs besoins corporels. L'ascétisme, sous toutes ses formes, est en effet recherché. La tendance des groupes ayant adopté une philosophie de pureté à s'isoler des autres dans le but d'éviter toute fréquentation fait en sorte qu'ils choisissent souvent des coins isolés pour s'établir. Il arrive qu'ils recherchent des lieux de plus en plus isolés géographiquement pour se protéger du contact avec l'autre. De même, ils valoriseront l'autosuffisance et l'autarcie est souvent la règle (Ibid.).

La question de recherche qui se dégage à la suite de cette contextualisation théorique vise à comprendre le rôle joué par trois éléments, soit a) le discours du protestantisme conservateur sur la punition corporelle des enfants - en ce que ce discours caractérise l'univers symbolique de l'Église étudiée, b) le type de leadership exercé par le dirigeant du groupe et c) les particularités du type de fonctionnement groupal, sur l'évolution de l'Église baptiste de Windsor, évolution vue comme ayant favorisé le recours à une forme excessive de punition corporelle des enfants.

\section{Méthodologie}

Afin de répondre à la question de recherche posée, une méthodologie de nature qualitative à été privilégiée, se basant principalement sur l'analyse documentaire à laquelle des entretiens avec des acteurs clés ont été ajoutés. L'échantillon documentaire a d'abord été constitué à partir d'une centaine de documents, dont 60 documents légaux: 37 provenant de la Cour Supérieure du district de Saint-François (chambre criminelle et pénale ainsi que chambre civile, Tribunal de la jeunesse, Bureau du Protonotaire) et 23 provenant d'organismes publics et parapublics (ministère de la Justice, Office des services de garde à l'enfance, Direction du fichier central des entreprises, Division de l'enregistrement de Richmond et Revenu Canada). Puis s'y sont ajoutés tous les articles de journaux publiés au Québec entre le 19 avril 1985 et le 15 mars 1990 au sujet de cette affaire, ainsi que deux documentaires réalisés par la Société Radio-Canada (12 avril 1985 et 28 octobre 
$\left.1986^{3}\right)$. De plus, les témoignages de plusieurs anciens membres ont été étudiés et des entretiens avec cinq acteurs clés dont l'inspecteur de police qui a mené l'enquête sur cette congrégation religieuse, un pasteur ayant connu celui à la tête de l'ÉBW, un ancien membre du groupe et un fonctionnaire de la municipalité de Windsor ont complété la collecte de données et permis une triangulation des sources d'information. Deux voyages sur place ont également été faits afin de situer l'environnement où les événements se sont déroulés.

Quoique la reconstruction historique effectuée couvre douze ans (Pacheco, 2002), seules les huit années d'existence de l'Église baptiste de Windsor (1978-1986) seront résumées et analysées dans le présent article. Les informations pertinentes au sujet du recours à la punition corporelle ont été classées en sous-thèmes comprenant, par exemple, les types de châtiments, leur fréquence, leur gravité (nombre de coups et présence ou non de rougeur, de marques ou de lésions), les personnes administrant les punitions, ou encore l'âge des enfants au moment des premières punitions corporelles. Ensuite, ces données ont été croisées aux informations obtenues auprès des personnes-ressources afin de les valider. Puis, elles ont été comparées aux caractéristiques du groupe à chacune des périodes étudiées afin de mieux comprendre comment le recours à la punition corporelle a pu donner lieu à des sévices physiques. Enfin, les résultats des analyses de chacune des étapes ont été croisés avec les données provenant d'autres sources afin de limiter les effets de biais qu'une telle étude comporte inévitablement.

Cette recherche comporte, comme toute recherche du genre, des limites liées à la valeur de l'information recueillie. Bien que bon nombre de documents proviennent de sources légales, et donc constituent des documents de grande qualité, d'autres proviennent d'entretiens et de témoignages d'anciens membres du groupe dont on sait que le discours peut comporter un biais en raison de la tendance à privilégier une vision qui diminue la responsabilité personnelle au profit de celle du dirigeant du groupe. Afin de diminuer l'effet possible d'un tel biais, les informations provenant d'anciens membres, lorsqu'elles consistaient à rejeter la faute sur autrui, n'ont été utilisées que lorsqu'elles trouvaient confirmation dans une autre source de données.

3. Les auteures remercient l'organisme Info-Secte pour leur avoir donné accès aux documents audiovisuels de la Société Radio-Canada et aux actes légaux de constitution de l'Église baptiste de Windsor. 


\section{Résumé historique}

Première période: de la fondation à la scission (1978-1980)

Le pasteur

Né en 1947, le pasteur $\mathrm{X}^{4}$ était un ancien résident de Windsor, petite municipalité de quelque 5000 habitants du sud-est du Québec. Au milieu des années 1970, il adhéra, tout comme un de ses frères, à l'Église baptiste évangélique de Lennoxville, et peu de temps après, il entreprit une formation pour devenir pasteur dans une école théologique située à Danville ${ }^{5}$. D'après les témoignages d'ex-membres de son Église, c'était un homme gentil, dévoué et doté d'une personnalité chaleureuse. Il amena d'ailleurs un bon nombre de convertis à l'Église baptiste évangélique de Lennoxville, ce qui poussa d'ailleurs cette congrégation à l'appuyer dans son projet de fonder une Église à Windsor.

\section{La fondation de la congrégation de Windsor}

Sur le plan administratif, l'Église baptiste de Windsor figurait comme une corporation indépendante ne faisant partie d'aucun regroupement religieux ni association. Son seul dirigeant a été le pasteur $\mathrm{X}$, assisté de quatre administrateurs. Elle avait été mise sur pied grâce à l'aide de l'Église baptiste évangélique de Lennoxville, ce qui conférait à cette dernière un droit de regard quant à la manière dont l'Église était gérée, tant du point de vue financier que doctrinaire ${ }^{6}$. L'Église de Lennoxville acquit en effet deux immeubles à Windsor et les prêta au pasteur $\mathrm{X}$ pour lui servir de temple et de presbytère. L'Église de Windsor commença à $y$ fonctionner officiellement le 6 juillet 1978. Vu son succès en termes de nombre de nouveaux membres, l'Église baptiste évangélique de Lennoxville lui céda les immeubles qu'elle occupait le 15 mai 1979.

4. La décision de taire le nom du pasteur ayant dirigé l'Église baptiste de Windsor, qui sera désigné par la lettre $\mathrm{X}$, a été prise afin d'éviter de participer à sa stigmatisation possible.

5. L'école de Danville n'a existé que pendant une année. D’après le pasteur interviewé, qui y a étudié en même temps que X, ce dernier n'a suivi aucune autre formation pour devenir pasteur. Cela est peu fréquent, la plupart des pasteurs devant suivre des études de plusieurs années, incluant des stages.

6. Il convient de souligner que, même si les Églises protestantes n'ont pas d'organisme recteur et qu'en ce sens elles sont indépendantes, elles font souvent partie d'associations confessionnelles. De façon non officielle, elles collaborent aussi entre elles, la forme la plus répandue de collaboration étant l'invitation faite aux pasteurs d'autres congrégations à prêcher dans son église. 
Désignée d'abord comme l'Église baptiste évangélique de Windsor (ÉBÉW), elle connut une croissance fulgurante et comptait en 1980, seulement deux années après sa fondation, environ 70 membres adultes. Bien qu'une trentaine d'entre eux provenaient de la congrégation de Lennoxville, les autres étaient des nouveaux convertis. Il s'agissait surtout de jeunes couples avec des enfants, pour la plupart des gens à faible revenu, soit des ouvriers non qualifiés, des personnes travaillant dans les services et des gens qui vivaient des prestations de l'aide sociale.

\section{Les normes}

L'Église souscrivait à la vision conservatrice selon laquelle l'homme, par droit divin, exerce l'autorité familiale sur l'épouse et les enfants qui lui doivent une obéissance absolue. On encourageait d'ailleurs les couples à avoir des enfants et on les exhortait à les éduquer de manière conforme aux interprétations bibliques conservatrices. Dans un tel schème, l'éducation des enfants revient au père, qui a l'obligation, devant Dieu, de les éduquer dans la foi et de les corriger quand ils s'éloignent de ce qui est considéré comme le bon chemin. L'injonction à châtier physiquement les enfants faisait d'ailleurs souvent partie des prédications du pasteur, qui recommandait la punition corporelle, souvent désignée par le vocable «correction», comme mesure disciplinaire destinée aux enfants dès l'âge de un an. Au cours de cette première période, aucune règle ne fut instituée en ce qui concerne l'administration des punitions, chaque famille décidant quelles conduites donnaient lieu à une punition. De même, bien que la punition corporelle était certes recommandée activement lors des prédications, la façon de l'administrer ne faisait pas l'objet de directives précises. L'utilisation d'un instrument, quoique suggérée, n'était pas obligatoire et c'était davantage la fessée qui était utilisée comme mesure disciplinaire.

Par ailleurs, il appert que très peu d'aspects de la vie quotidienne étaient régis de façon formelle au cours de cette première période. Hormis l'interdiction de boire de l'alcool et l'obligation de payer la dîme, fixée à $10 \%$ du revenu, aucune autre règle de conduite n'était formellement en vigueur. Mais, des us et coutumes s'imposèrent néanmoins rapidement. En effet, les nouveaux membres calquaient leurs conduites sur celles des membres plus anciens, recherchant leur approbation, alors que ces derniers s'efforçaient de suivre toutes les recommandations du pasteur au pied de la lettre. 


\section{La scission}

Malgré le succès de l'Église en termes de recrutement, plusieurs différends, tous liés à l'interprétation de la Bible, éclatèrent entre le pasteur et certains membres au cours de l'année 1980. Selon les témoignages entendus, le pasteur avait une position très rigide au sujet de l'interprétation des versets bibliques; il n'acceptait pas les discussions au sujet de ses interprétations, d'ailleurs vues comme des interprétations à orientation fondamentaliste. Le pasteur tenait à ce que les membres mettent intégralement en application certains versets de la Bible dans leur vie quotidienne de la façon la plus littérale possible afin, disait-il, de ne pas trahir la volonté divine. Cela s'appliquait, entre autres, aux versets relatifs aux châtiments corporels. Une scission eut lieu à la suite de la décision du pasteur de s'engager dans la formation de jeunes qui se préparaient à devenir pasteurs. Une partie des membres, soit environ la moitié de l'assemblée dont la majorité des membres de longue date ayant appartenu auparavant à l'Église de Lennoxville, quittèrent l'Église de Windsor. D'ailleurs, selon les informations livrées par Sylvain ${ }^{7}$, ancien membre du groupe, plusieurs communautés baptistes de la région coupèrent alors leurs liens avec la congrégation de Windsor et le pasteur $\mathrm{X}$ considérant qu'il était devenu trop rigide et intransigeant. C'est ainsi que l'Église de Windsor se trouva isolée des autres groupes baptistes de la région.

\section{Deuxième période: essor de l'Église baptiste de Windsor (1981-1982)}

La rupture avec l'Église baptiste évangélique de Lennoxville laissa la gestion de l'Église, appelée désormais Église baptiste de Windsor (ÉBW), entre les mains de $\mathrm{X}$, de son frère, d'un pasteur adjoint et des quatre administrateurs. Il ne restait qu'une quarantaine de fidèles, mais deux ans plus tard, grâce à un prosélytisme actif, l’Église avait entre 120 et 150 membres $^{8}$. Le fait d'avoir autant de nouveaux membres obligea le pasteur à rendre explicites des règles jusqu'alors informelles, ce qui les rendit d'ailleurs plus rigides encore. Nombre de conduites

7. Nom fictif.

8. Nus n'avons pas réussi à connaitre le nombre exact de membres. Nous faisons donc une approximation à partir de différents témoignages. 
auparavant seulement déconseillées verbalement furent dorénavant clairement prohibées. De plus, des directives interdisaient de nouvelles conduites, par exemple, regarder la télévision, acheter des objets décoratifs, superflus ou encore manger des sucreries. Bien que la fréquentation de personnes hors de l'Église ne fût pas prohibée, cela était mal vu sauf en ce qui concerne le prosélytisme. La multiplication des règles au fur et à mesure de la croissance du groupe donna aussi naissance à plusieurs mécanismes de contrôle: le pasteur, aidé d'un petit cercle de proches dont son frère et les administrateurs, exerçait une surveillance étroite des conduites des membres. Un climat de délation s'ensuivit auquel le pasteur donna des suites en menaçant d'expulsion et de damnation éternelle les membres dont les manquements lui étaient signalés.

Entre prosélytisme, travail bénévole et services religieux, le temps alloué aux activités non liées à l'Église se réduisit considérablement, conduisant tout naturellement à une forme d'isolement du monde extérieur. Cet isolement fut d'autant plus accentué que le pasteur craignait que les membres de l'Église soient contaminés par le contact avec tous ceux qui étaient extérieurs au groupe, qu'il désignait tantôt comme des «païens» tantôt, avec mépris, comme des «pourceaux». La crainte de la contamination s'amplifia au point où le pasteur interdit éventuellement tout contact avec les gens extérieurs à la congrégation.

\section{Doctrine}

Le fondamentalisme du pasteur s'accentua au cours de la seconde période. Les règles de conduite devinrent aussi progressivement plus sévères, ce qui augmenta la difficulté à les faire respecter, notamment par les enfants, qui furent l'objet de plus de sévérité et donc de corrections physiques plus fréquentes. En outre, le pasteur décréta que désormais les corrections physiques devaient obligatoirement être administrées avec un objet. Une grande attention fut ainsi portée à la façon d'éduquer les enfants et plus spécifiquement de les punir. Par exemple, le pasteur projeta des diapositives illustrant la manière correcte d'administrer les punitions corporelles; dans ce cas précis, cinq coups frappés sur les fesses dénudées avec une verge, soit un bâton en bois. Avec la fondation de l'école et de la garderie de l'Église, ces normes furent cependant modifiées. 


\section{Création d'une garderie et d'une école}

Afin de soustraire les enfants de l'influence - vue comme corruptrice - du monde extérieur, une garderie et une école furent fondées en 1981. Tous les enfants devaient les fréquenter et ce fut le pasteur qui en désigna les moniteurs et les enseignants parmi les membres de l'Église. L'attention désormais portée à la discipline des enfants s'accrut de façon significative. Un désir explicite de faire d'eux des enfants modèles, voire parfaits, c'est-à-dire dénués de bon nombre de leurs caractéristiques enfantines, guidait dorénavant la façon d'interagir avec eux. L'objectif maintes fois répété d'obliger l'enfant à renoncer à sa nature perverse s'amalgamant dès lors à une représentation idéalisée d'un enfant parfaitement pur. À cet effet, les objectifs fixés par le programme scolaire reflétaient un idéal de perfection, par exemple, la note de passage fut fixée à $80 \%$ et les enfants devaient maintenir une moyenne supérieure à cette note. De telles exigences eurent pour conséquence que certains enfants furent rapidement identifiés comme particulièrement pervers et impurs, ce qui justifia un recours plus fréquent à la punition corporelle à leur endroit. Puisque tout avait été mis en œuvre pour soustraire les enfants des influences néfastes, toute performance, qu'elle fut scolaire ou comportementale, jugée insatisfaisante était interprétée comme un signe de malveillance chez l'enfant, plus précisément comme un signe de l'œuvre de Satan dans son âme. Cette interprétation justifiait aux yeux du pasteur le recours à une sévérité accrue dans les punitions infligées. De fait, tous les élèves étaient dorénavant soumis à un code de conduite très strict; par exemple, dès la réception de trois billets de l'enseignant rapportant un manquement de l'enfant, son père devait lui administrer cinq coups de verge.

Afin de s'assurer que les punitions seraient réellement administrées, le pasteur décida que désormais elles auraient lieu à l'école, les parents étant avertis la veille de la punition prévue. Un instrument particulier fut fabriqué pour infliger ces punitions, soit une sorte de férule ou de baguette en bois de chêne de $45 \mathrm{~cm}$ de longueur, par $6 \mathrm{~cm}$ de largeur et $2 \mathrm{~cm}$ d'épaisseur. Bien que les enfants de l'école furent les premiers à être ainsi punis par le pasteur ou l'un des enseignants, les châtiments s'étendirent rapidement aussi aux petits de plus de un an fréquentant la garderie. Tout ce qui pouvait être interprété comme un signe de rébellion ou de désobéissance chez eux faisait l'objet de corrections physiques, que ce soit des manquements dans l'apprentissage du con- 
trôle des sphincters ou l'incapacité de dormir à l'heure de la sieste. Chez les enfants fréquentant l'école, le fait de regarder un autre enfant, d'avoir une note inférieure à la moyenne ou encore de demander d'aller aux toilettes hors du temps prévu à cette fin étaient considérés comme des conduites méritant un châtiment corporel.

\section{Troisième période: l'isolement du groupe (1983-1984)}

À partir de 1983, le nombre de membres de l'ÉBW cessa d'augmenter. D’abord, parce que l'Église était désormais perçue comme étant particulièrement rigide, mais aussi parce que l'effort mis dans le prosélytisme diminua considérablement. Certaines stratégies de recrutement utilisées par l'ÉBW avaient d'ailleurs été contestées, notamment par des représentants de l'Église catholique, en particulier la pratique consistant à organiser des spectacles pour enfants fortement publicisés dont l'objectif principal était la conversion des parents qui y accompagnaient leurs enfants. En outre, l'ÉBW fut l'objet de rumeurs concernant certaines transactions financières faites par le pasteur à son profit ou à celui de l'Église. La représentation sociale du pasteur, telle qu'elle se dégage des témoignages entendus, changea d'ailleurs au cours de 1983-1984 et désormais fut plutôt négative.

L'importance du rôle joué par le pasteur dans la vie quotidienne des membres augmenta considérablement à partir de 1983. Par exemple, il négociait dorénavant les primes d'assurance automobile des membres, faisait effectuer les réparations de véhicules dans un garage qui lui appartenait, choisissait et distribuait les denrées alimentaires aux membres. De plus, de nombreux membres travaillaient pour des entreprises qui lui appartenaient. Il caressait aussi le projet de construire un domaine immobilier autosuffisant où seuls les membres de l'ÉBW pourraient habiter. Pour amasser les fonds nécessaires à la réalisation de ce projet, les membres durent multiplier les dons, contracter des emprunts et même vendre leurs biens. En septembre 1984, l'Église acquit un terrain que les membres défrichèrent pour commencer la construction du domaine.

\section{L'émissaire de Dieu}

Désormais le pasteur se décrivait comme l'émissaire choisi par Dieu pour lutter contre les forces du mal. Cette vision de lui-même le conduisit à se déclarer infaillible. Il exigeait aussi une soumission absolue 
et enjoignait les membres à «entrer dans la joie de leur maître en étant des fidèles esclaves ${ }^{9} »$. Il exigeait toujours plus de travail des membres, mais aussi plus d'argent de leur part, arguant que la Bible dictait que tout ce qui leur appartenait lui appartenait. Du haut de la chaire, il traitait les membres de sa congrégation d'avares et d'hypocrites et les ridiculisait devant l'assemblée s'ils tentaient alors de se défendre. Bien qu'il était difficile pour nombre de membres de quitter l'ÉBW, car tout leur avoir avait été remis à l'Église, qui l'avait à son tour investi dans la réalisation du domaine, plusieurs partirent néanmoins, certains parce qu'ils se sentaient épuisés et exploités ${ }^{10}$ par le pasteur. D'autres invoquaient le traitement infligé aux enfants. Il est à remarquer que ce ne furent que des hommes qui invoquèrent cette dernière raison. En quittant l'Église, quelques-uns laissaient aussi leurs épouses et leurs enfants.

\section{L'intensification des châtiments corporels}

Le discours relatif à la punition corporelle se durcit au cours de cette troisième période, soit en 1983 et 1984. L'accent étant désormais mis sur le besoin de corriger les enfants dès leur plus jeune âge pour exorciser l'emprise de Satan. Le pasteur était ainsi d'avis que plus les enfants vieillissaient, plus il était difficile de redresser leur nature et d'y chasser le mal. Il ordonna en conséquence d'administrer des punitions aux enfants de moins de un an. Les nourrissons étaient dorénavant frappés à la garderie s'ils ne dormaient pas aux heures prévues ou encore s'ils pleuraient. Les cinq coups de verge jusqu'alors réglementaires furent abandonnés: pour tous les enfants, le nombre de coups était désormais laissé à la discrétion de l'adulte qui imposait la punition. De plus, l'attitude de l'enfant pendant sa punition comptait dans le nombre de coups administrés; les enfants qui tentaient de se soustraire au châtiment en couvrant leurs fesses de leurs mains, par exemple, étaient frappés jusqu'à ce qu'ils adoptent l'attitude de soumission attendue.

Une autre modification apparue en 1983 consistait en l'introduction de corrections publiques. C'était le père qui devait alors administrer les coups de verge à son enfant pendant les services religieux. Si le père

9. Extrait d'un sermon donné par X qui apparaît dans le documentaire de Le Point du 12 avril 1985 (Société Radio-Canada, 1985).

10. Les anciens membres qui ont entrepris des litiges civils contre le pasteur ont qualifié d'exploitation le traitement dont ils auraient été l'objet de sa part. 
n'était pas présent, le pasteur le remplaçait. En général, la punition avait été décidée d'avance, mais parfois elle découlait d'un manquement à la discipline pendant l'office même. Le nombre de coups pendant ces séances publiques était, en général, plus élevé qu'à l'école, pouvant aller jusqu'à 50. Ainsi, il n'était pas rare que les punitions corporelles administrées laissent des marques. Les punitions dites légères laissaient des petites marques rouges en forme de lignes, mais les punitions plus sévères laissaient des bleuissements de la longueur de la férule sur le corps de l'enfant alors que, lorsque l'enfant avait été corrigé plusieurs fois sur une courte période, des marques noires apparaissaient sur sa peau.

Les témoignages des membres de l'ÉBW devant la Direction de la protection de la jeunesse de l'Estrie, au Tribunal de la Jeunesse ou aux médias permettent d'établir que les membres acceptaient la conviction de leur pasteur selon laquelle les punitions qu'ils administraient à leurs enfants étaient nécessaires pour assurer leur salut. Certains avouèrent avoir parfois été choqués par leur sévérité, mais ajoutèrent que cela n'infléchissait pas leur conviction que le fait d'administrer de telles punitions corporelles était une preuve de leur amour envers leurs enfants et de leur obéissance à la volonté de Dieu. Bien que certains disent avoir été conscients que ces punitions auraient été jugées excessives par les gens extérieurs à leur groupe - d'aucuns savaient même qu'elles pouvaient être jugées criminelles, notamment un membre qui était policier à la Sûreté du Québec - cela ne remettait pas en cause leur conviction du bien-fondé d'administrer de tels châtiments. Il a aussi été avancé, lors de ces témoignages, que la crainte d'être incompris, voir traduits devant un tribunal, était faible étant donné que les parents et surtout leurs enfants avaient très peu de contacts avec des gens hors de l'Église.

Quatrième période: vers la dissolution de l'Église (1985-1986)

Malgré l'isolement du groupe, des rumeurs commencèrent à circuler hors du groupe au sujet du traitement des enfants qui y résidaient. Ce sont d'anciens membres qui, bien qu'ils n'eurent toutefois jamais précisé la nature des corrections physiques administrées aux enfants, possiblement par crainte d'en être tenus responsables du temps de leur adhésion à l'Église, contribuèrent néanmoins à en faire connaître l'existence. Les autorités policières prirent connaissance de ces rumeurs dès 1984, mais ce ne fut qu'en janvier 1985 qu'une enquête fut ouverte, lorsqu'une femme dont le bébé de cinq mois avait été battu à la garderie, se laissa 
convaincre par son mari - qui avait déjà laissé le groupe - de déposer une plainte pour voies de fait contre le pasteur. Alarmée par les pratiques punitives décrites par la plaignante, la police municipale de Windsor élargit alors son enquête et fit un signalement à la Direction de la protection de la jeunesse de l'Estrie (DPJE) afin qu'elle enquête à son tour sur une situation potentielle de maltraitance généralisée à l'ÉBW.

\section{L'enquête de la police et celle de la DPJE}

L'enquête de la police se heurta, dans un premier temps, au silence non seulement des membres de l'ÉBW, mais aussi des anciens membres. D'un côté, les membres cherchèrent d'abord à protéger leur mode de vie, considérant qu'ils ne devaient répondre de leurs actions qu'à Dieu. D'un autre côté, les anciens membres, quant à eux, craignaient de s'inculper et d'inculper leurs proches s'ils décrivaient comment les enfants y étaient traités. Néanmoins, après deux mois d'enquête, la police signala une dizaine de cas additionnels à la DPJE. Le directeur de la DPJE de l'époque décida de tenir compte du contexte sectaire dans lequel la maltraitance avait été signalée. Il planifia ainsi une intervention globale plutôt que de répartir les cas à des délégués sans tenir compte de l'ensemble du dossier. De concert avec le Centre des services sociaux de l'Estrie (CSSE), il forma une équipe constituée de travailleurs sociaux, d'avocats, de psychologues et d'autres experts afin d'aborder de façon optimale les différents aspects du signalement. Dès le mois de mars 1985, des travailleuses sociales commencèrent à contacter des parents de l'ÉBW pour évaluer la situation de leurs enfants.

Le pasteur, qui n'avait pas été convoqué par la police lors de son enquête, ne se montra pas particulièrement inquiet. Il répétait aux membres de l'Église que la police et la DPJE ne pouvaient rien prouver contre eux et qu'elles allaient rapidement mettre fin à leurs enquêtes. Toutefois, les parents devaient recevoir les intervenants sociaux de crainte de se voir retirer la garde de leurs enfants. À cet égard, le pasteur ordonna aux membres de mentir au sujet des punitions corporelles et décréta leur arrêt temporaire de peur que la DPJE fasse examiner les enfants et qu'on découvre des marques sur eux. Pour leur part, les travailleurs sociaux avaient été avisés de faire preuve d'une grande discrétion dans leur enquête afin de ne pas provoquer une situation qui mettrait les enfants à risque d'être punis pour avoir dévoilé le type de châtiments qui leur était administré. 


\section{Le dévoilement des agissements de l'ÉBW}

Le 12 avril 1985, l'émission Le Point de la Société Radio-Canada diffusa un reportage télévisé sur l'ÉBW, basé en grande partie sur des témoignages d'ex-membres. La vie des membres y était décrite comme étant sous le contrôle du pasteur et les punitions corporelles administrées aux enfants furent présentées sous cet angle. En outre, deux fillettes âgées de cinq et sept ans ainsi que deux adolescentes étaient interviewées au sujet des punitions qu'elles y avaient reçues. Le reportage attira l'attention de la presse régionale qui dédia un nombre important d'articles à l'affaire. Ce reportage, dans lequel le directeur de la DPJE est aussi interviewé, semble avoir conduit la DPJE à agir avec célérité. Le 19 avril 1985, le directeur de la DPJE déclara à la presse qu'une vingtaine des cas seraient soumis au Tribunal de la jeunesse car: "[l'enquête] porte sur des cas de violence excessive. Battre un enfant pendant une demiheure pour une vétille, ce n'est pas raisonnable ${ }^{11} »$. Il aborda aussi la situation irrégulière des établissements scolaires du groupe qui n'avaient pas de permis. L'Office des services de garde à l'enfance (OSGE) envoya, en conséquence, des inspecteurs à la garderie, mais on leur refusa l'accès. Les activités de garde cessèrent quelques jours plus tard et l'OSGE ferma son dossier.

Si le pasteur apparut serein devant ses membres en ce qui concernait l'enquête policière et celle de la DPJE, il fut plus inquiet à la suite de la parution du documentaire de la Société Radio-Canada et des articles sur l'ÉBW. Il décréta que la porte du temple devait dorénavant demeurer verrouillée, même pendant les services afin d'empêcher que des journalistes puissent y filmer ses prédications. De plus, il interdit aux membres de leur adresser la parole et ordonna que cesse tout contact qui puisse encore exister avec des gens hors de l'Église, y compris avec les anciens membres, qui furent alors désignés comme des espions et des déserteurs. Il soutint d'ailleurs que toute intervention contre l'Église était le fruit d'un complot ourdi par les anciens membres, désormais corrompus par Satan, et décidés à détruire sa congrégation. Il présenta aussi les intervenants de la DPJE et de l'OSGE, ainsi que les journalistes comme des pions utilisés par Satan agissant à travers les déserteurs, terme désignant

11. Ces propos ont été publiés par le quotidien de Sherbrooke La Tribune le 20 avril 1985 dans l'article de Gilles Dallaire intitulé: «Au sein de la communauté baptiste de Windsor. Le Tribunal de la Jeunesse pourrait se pencher sur des cas de sévices». Larticle en question était le $4^{e}$ d'une série de quatre sur l'ÉBW écrite par Gilles Dallaire. 
les ex-membres. En outre, les interventions des médias ainsi que celles des agents de contrôle social étaient décrites par le pasteur comme autant d'épreuves voulues par Dieu pour mettre leur foi à l'épreuve.

Bien que les membres répétaient d'abord les paroles de leur pasteur dans leurs propres discours, au fur et à mesure que l'enquête de la DPJE avançait, la peur de perdre la garde de leurs enfants s'imposa et en conduit plusieurs à s'exprimer de façon plus personnelle, prenant une distance par rapport aux enseignements du pasteur au sujet de la punition corporelle. D'ailleurs, quelques membres quittèrent la congrégation pour bien marquer leur distanciation par rapport aux pratiques éducatives qu'ils avaient jusqu'à récemment eux-mêmes utilisées. Certains découvrirent, à travers la représentation que les médias faisaient de leur groupe, qu'ils avaient été totalement soumis à l'autorité de leur pasteur, ce qui les amena à remettre en question leur affiliation à l'ÉBW. Le pasteur, quant à lui, quitta Windsor et ne se rendit plus au temple que pour y diriger les services. Il y insultait alors les membres en les qualifiant de délateurs. Bien qu'il eût ordonné l'arrêt des punitions corporelles depuis le début des enquêtes, il corrigea lui-même quelques enfants de manière très sévère durant cette période. À titre d'exemple, un enfant de six ans fut battu à trois reprises au cours d'une même semaine durant les services religieux.

\section{La dissolution de l'ÉBW}

En décembre 1985, le nombre de signalements retenus à la DPJE était de 84. Ils concernaient surtout la pratique de châtiments corporels vus comme susceptibles de nuire à la sécurité des enfants selon l'article 38 (e) de la Loi sur la protection de la jeunesse. En outre, comme l'isolement extrême dans lequel les enfants vivaient fut également vu comme susceptible de compromettre leur développement, tous les enfants du groupe furent placés sous la protection de la loi même s'il n'existait pas de preuves qu'ils aient été battus. Afin d'éviter la judiciarisation des cas de leurs enfants, on proposa aux parents de s'engager à modifier leur façon de discipliner leurs enfants et à les réintégrer dans la société. Certains enfants furent recommandés pour un suivi psychologique puisqu'on évalua chez eux des difficultés peu susceptibles d'être corrigées par les mesures sociales et familiales prises. La majorité des parents acceptèrent ces mesures, dites volontaires dans le jargon de la loi. Les cas de sept enfants durent toutefois être acheminés au Tribunal de la 
jeunesse parce que leurs parents refusaient les mesures volontaires proposées par les intervenants sociaux.

Bien que l'Office des services de garde à l'enfance (OSGE) eut fermé son dossier en 1985 croyant que le service de garde de l'ÉBW était fermé, les agents de cet organisme décidèrent, en apprenant que la garderie continuait de fonctionner, de procéder à une fermeture officielle en janvier 1986, en vertu des dispositions de l'article 3 de la Loi sur les services de garde à l'enfance. En prévision d'un possible refus de la part des membres de l'ÉBW d'accepter cette fermeture, l'OSGE sollicita la collaboration de la police municipale de Windsor et de la Sûreté du Québec pour leur faire évacuer les lieux. La DPJE envoya aussi des intervenants en prévision de cas où des enfants devraient être pris en charge. Aucun incident regrettable n'eut cependant lieu. Le pasteur n'était pas sur place le jour de la fermeture de la garderie et peu après, il s'installa définitivement dans une ferme située dans une autre région, depuis laquelle il géra dorénavant sa congrégation. Ce déménagement fut vécu comme un abandon par bon nombre de membres de l'ÉBW, ce qui diminua considérablement son autorité sur eux et facilita grandement le travail des intervenants sociaux et contribua à la dissolution du groupe.

L'intervention de l'OSGE visait uniquement la fermeture de la garderie, mais elle signa de fait la fin des activités régulières de l'Église. Si l'enquête de la DPJE et sa médiatisation avaient déjà provoqué un bon nombre de départs, le fait que l'intervention de l'OSGE se fit de concert avec un important dispositif policier eut un effet certain sur les membres restants de l'Église. Ainsi, tout au cours de l'année 1986, des membres quittèrent l'Église, à tel point qu'en décembre de cette annéelà seules quelques personnes continuaient à se réunir autour du pasteur $\mathrm{X}$. Cependant, il est à signaler qu'à ce moment-là, il avait quitté Windsor définitivement et que les immeubles de l'Église avaient été mis en vente.

Le Bureau du procureur général du Québec accorda une immunité de poursuites judiciaires à tous les membres de l'Église baptiste de Windsor à l'exception de $\mathrm{X}$. De plus, il eut à faire face à deux poursuites civiles engagées par des ex-membres en plus des accusations criminelles de voies de fait auprès de six enfants de l'ÉBW ${ }^{12}$. Il fut condamné

12. Le mandat d'arrestation contre X, émis le 8 octobre 1987 , contenait huit chefs d'accusation en vertu de l'article 245 du Code criminel annoté et lois connexes (Canada, 1987: 224). À l'époque, cet article concernait les voies de fait avec infliction de lésions corporelles et les infractions étaient passibles de 10 ans d'emprisonnement. 
le 15 mars 1990 à une peine d'emprisonnement de 90 jours à être purgée de façon discontinue et à une probation de deux ans pendant lesquels il lui était interdit d'occuper tout poste au sein d'une congrégation religieuse. De plus, le juge André Fauteux fit jurisprudence dans son jugement concernant une des causes entendues devant le Tribunal de la jeunesse de l'Estrie relative à une adolescente déficiente qui fut victime de sévices physiques et sexuels dans le groupe. Le juge souleva la question de la prédominance des dispositifs de la Charte des droits et libertés de la personne concernant la liberté religieuse sur ceux de la Loi sur la protection de la jeunesse. Il conclut que les dispositifs de la Loi sur la protection de la jeunesse devaient prédominer, et ce, indépendamment des articles 1 et 2 de la Charte des droits et libertés de la personne:

La liberté de religion n'est pas absolue. Elle n'autorise personne à refuser à l'enfant le libre exercice de son droit à la protection, à la vie, au respect de sa personne, à son développement mental et affectif et à sa sécurité. La liberté de religion n'autorise non plus personne à enfreindre les règles de droit qui assurent à l'enfant le libre exercice de ses propres droits fondamentaux. (Recueils de jurisprudence du Québec, 1986: 2712)

\section{Discussion}

La situation des enfants connut une détérioration certaine au cours de l'histoire de l'Église baptiste de Windsor, comme la reconstruction historique permet de saisir d'emblée. Le découpage en différentes périodes permet de constater que le recours au châtiment corporel comme principal mode de punition s'y est systématisé, intensifié et aggravé en étroite relation avec les transformations subies par l'Église à chacune des quatre périodes isolées. De plus, le recours au châtiment corporel s'est peu à peu étendu à l'ensemble des enfants, des nourrissons aux adolescents. À la fondation de l'ÉBW, les châtiments corporels étaient d'abord administrés seulement par les parents et sans instrument ni codification imposés. Puis, l'utilisation d'un bâton de chêne fut requise et les punitions furent aussi administrées par des personnes autres que les parents. Progressivement le recours aux punitions devint quotidien et s'étendit aux nourrissons. Enfin, les punitions furent administrées même publiquement et prescrites pour des fautes qui, en fait, ne constituaient que des manifestations normales propres à l'âge des enfants. La présente section présentera une discussion des éléments et 
des dimensions qui semblent avoir joué un rôle dans l'escalade des punitions corporelles vers des mauvais traitements observés à l'Église baptiste de Windsor.

D'abord, il y a lieu de noter que la situation de maltraitance qui s'est développée à l'ÉBW a été favorisée, dès sa fondation, par son orientation protestante conservatrice puisque la doctrine recommande non seulement le recours à la punition corporelle, mais de plus l'utilisation d'un instrument pour l'administrer. Comme l'observent Bartkowski et Ellison (1995) ainsi qu'Ellison (1996), l'attitude favorable à la punition corporelle et l'utilisation d'un objet pour l'administrer constituent la combinaison de facteurs les plus susceptibles de mener à une situation de sévices physiques des enfants. Bien que la punition corporelle sans instrument, la simple fessée avec la main, ait été vue jusqu'à la scission comme acceptable par l'ensemble de la congrégation, la scission a donné lieu à un changement d'attitude du pasteur à l'égard des normes de conduite prescrites et tout particulièrement de l'attitude éducative à adopter avec les enfants. Son interprétation fondamentaliste de la Bible, après la scission, a conduit le pasteur à une application littérale des versets bibliques, ce qui entraîna une codification du recours au châtiment corporel, notamment par l'introduction d'un instrument pour l'administrer et d'un protocole pour déterminer non seulement les fautes donnant lieu au châtiment, mais aussi le nombre de coups à donner et la façon exacte d'administrer le châtiment.

Le pasteur invoquait souvent son désir de faire des enfants de sa congrégation de bons chrétiens pour justifier le recours à la punition corporelle. À ce sujet, plusieurs auteurs (Illick, 1974; Walzer, 1974; El Mountacir, 1994; Casoni, 2000) soulignent que les enfants sont fréquemment surinvestis par les groupes religieux minoritaires, qui voient en eux leur seule chance de pérennité. Il se dégage des données colligées que cela constituait une préoccupation certaine du pasteur, qui avait la ferme volonté de former la relève. Willaime (1992) remarque d'ailleurs que l'absence de continuité confessionnelle associée à la naissance ou conférée d'emblée aux nourrissons rend la transmission de la foi aux enfants un souci constant et un objectif impératif chez les Protestants.

Cet élément a certainement exercé un effet encore plus important d'abord à partir du moment où l'ÉBW a vu son membership fondre de moitié lors de la scission, puis à partir de la troisième période, soit en 1983-1984, lorsque l'Église a connu une chute additionnelle du nombre 
de ses membres due à la diminution de ses activités de prosélytisme et aux départs volontaires de membres. La tension entre la représentation négative des enfants, propre au protestantisme conservateur, et le besoin de les en sauver par la punition corporelle afin qu'ils perpétuent la foi de leurs parents a alors certainement été exacerbée, ce qui justifiait d'autant plus facilement l'intensification et la systématisation du recours à la punition corporelle aux yeux des membres. Il est à remarquer de plus que la codification de la punition corporelle introduite par le pasteur au cours de la deuxième période coïncida avec un plus grand isolement $\mathrm{du}$ groupe tant par rapport au monde extérieur que par rapport aux autres groupes baptistes de la région. Un tel isolement n'a pu que nuire à l'exercice du jugement des membres qui, placés devant des changements motivés par la lecture biblique fondamentalisme de leur pasteur, n'avaient plus l'occasion de se confronter aux opinions d'autrui lorsqu'ils s'interrogeaient sur les nouvelles pratiques introduites par celui-ci.

Tant que les efforts de prosélytisme ont été grands, les contacts avec des gens hors du groupe ont été fréquents. Leur regard sur les membres de l'ÉBW, sur leurs façons d'être et de se comporter avec les enfants, tel un miroir, pouvait ainsi jouer un rôle réflexif qui, à tout le moins, obligeait les membres à se situer par rapport à une norme différente, et dans ce cas-ci, à une norme qui souvent excluait même le recours à la punition corporelle. Toutefois, à partir du moment où non seulement les contacts avec les gens de l'extérieur du groupe ont été formellement interdits, mais que les activités de prosélytisme ont également diminué, soit à la troisième période, la fonction réflexive jouée par le regard de l'autre a cessé d'être opérante. Un membre en a fait l'observation d'ailleurs, notant qu'à partir du moment où ils ne vivaient plus qu'entre eux, soit à partir de la troisième période en 1983-1984, il ne s'était plus demandé que très rarement comment quelqu'un d'extérieur au groupe interpréterait leur façon de traiter les enfants. Un effet pernicieux paradoxal a même pu se produire dans le sens où plus les membres se sont trouvés isolés, plus ils ont pu être tentés de se conforter entre eux au sujet de leurs pratiques éducatives afin de calmer en eux tout doute ou toute inquiétude quant à leur bien-fondé.

Il est permis de penser que c'est justement la tendance à se positionner par rapport à une norme autre, représentée par le regard de l'autre sur soi, que le pasteur a cherché à fuir en condamnant les gens extérieurs au groupe comme sources de contamination menaçant la foi des 
membres. Il peut ainsi être avancé que le désir du pasteur d'éloigner les membres des autres peut être compris, dans ce sens, comme une tentative de mettre en échec la capacité de jugement individuel des membres, capacité plus susceptible de constituer une menace à son interprétation biblique si les membres pouvaient se comparer à des normes et à des points de vue autres, que s'ils ne se retrouvaient qu'entre eux. Le désir de protéger les membres de la contamination de leur âme était mis de l'avant comme argument justifiant l'interdiction de contact avec des personnes hors du groupe (Casoni, 2000). En outre, le pasteur décrivait ses fidèles comme spirituellement supérieurs non seulement aux nonmembres, mais aussi aux membres d'autres congrégations baptistes. Lors de ses prédications, il soulignait comment leur façon de vivre leur foi plaisait particulièrement à Dieu. À cet effet, il faut rappeler comment, dès la troisième période, soit en 1983-1984, le pasteur revendiquait l'infaillibilité et se disait être l'émissaire de Dieu pour lutter contre les forces du mal. Les membres participaient de facto à cette glorification de leur congrégation et à l'idéalisation de leur pasteur, s'accordant par association une part de cette dernière. En effet, à partir du moment où ils accordèrent à leur pasteur l'infaillibilité qu'il revendiquait, ils se lièrent à lui dans un rapport autoidéalisant réciproque où la grandeur attribuée au pasteur rejaillissait sur l'ensemble de la congrégation (Casoni, 2001).

D'ailleurs, les changements survenus au cours de l'histoire de l'ÉBW ont transformé graduellement le fonctionnement de cette congrégation en un fonctionnement d'allure sectaire. Au début, le groupe avait un fonctionnement assez souple caractérisé par des normes flexibles, un partage relatif de l'autorité et une certaine ouverture au monde extérieur. Six années plus tard toutefois, le groupe était engagé dans la réalisation d'un projet qui visait à l'établissement d'une véritable autarcie qui passait par la construction d'un domaine privé (Casoni, 2001). Dès lors, plusieurs membres se disaient prêts à braver les lois des hommes si elles contrevenaient à celles de Dieu telles qu'elles avaient été révélées au pasteur dans son interprétation biblique, ce qui témoigne d'une radicalisation et d'une distanciation non équivoques par rapport à la société plus large (Casoni, 2001; Casoni et Brunet, 2005). Au-delà du besoin groupal de simplement se différencier des non-membres par l'adoption d'un langage, de normes vestimentaires et de codes de conduite distincts, caractéristiques d'une philosophie de la séparation (comme ce fut le cas de la première à la deuxième période, soit de 1980 
à 1982), l'évolution de l'Église baptiste de Windsor conduisit les membres à adopter, dès 1983, une philosophie dite de pureté (Casoni, 2000). L'ÉBW en vint à concevoir le monde en termes de leur supériorité morale absolue, soit une caractéristique associée à l'adoption d'une philosophie de la pureté. L'ascétisme s'imposa et le pasteur, qui s'occupait des approvisionnements en vivres des membres, veillait à ce que les familles reçoivent le minimum de nourriture nécessaire pour vivre. Ainsi, le groupe a vraisemblablement subi non seulement l'effet des contraintes doctrinales propres au conservatisme protestant, mais, de plus, celles spécifiquement associées à la recherche de la pureté comme idéal spirituel tel que cela se rencontre dans les groupes qui souscrivent à une philosophie de la pureté. La pression groupale mise en œuvre afin d'atteindre les idéaux associés à la pureté spirituelle justifiant le fait de recourir à tous les excès répressifs mis en lumière par la reconstruction historique.

Le souci de se soustraire à la contamination attribuée au contact avec le monde extérieur a aussi conduit à une ségrégation croissante des enfants qui ont été presque entièrement soustraits au monde environnant, selon les témoignages entendus. Ceux-ci, surinvestis en tant que garants de la foi et du style de vie du groupe (Illick, 1974; Walzer, 1974; El Mountacir, 1994), mais aussi surveillés de près en raison du besoin doctrinal de redresser leur nature malsaine (Dobson, 1976, 1987), et du besoin idéologique de les débarrasser de leur impureté (Casoni, 2000), faisaient l'objet d'une attention vigilante afin que toutes les conduites ou les attitudes qui dérogeaient, ne serait-ce que minimalement, de l'idéal projeté sur eux étaient réprimées sévèrement. En même temps, l'impossibilité pour les enfants d'atteindre les idéaux fixés par le groupe mena à une escalade graduelle de la sévérité et de la fréquence des châtiments à leur encontre.

De plus, le processus manichéen de dichotomisation du monde en gens purs et impurs, caractéristique d'une philosophie de la pureté, correspond à des représentations absolument négatives, comme celle de la nature mauvaise de l'enfant d'un côté et, de l'autre, à des représentations qui leur sont totalement opposées, soit d'êtres purs, purgés des impulsions, des besoins et des caractéristiques corporelles habituelles de l'humain. Plus les premières représentations seront chargées de sens négatif, plus celles qui leur sont opposées seront idéalisées. Dans le cas présent, la représentation idéalisée était constituée par ce que visait la punition corporelle, soit de débarrasser complètement l'enfant du mal 
dont son âme était supposément habitée. Cette représentation idéalisée constituait ce à quoi les membres aspiraient dans leur traitement de l'enfant. Une quête d'absolu s'y dessinait qui a facilement pu motiver un parent à exiger toujours plus de son enfant tout en l'amenant, inévitablement, et de façon corollaire, à identifier toujours plus de comportements ou d'attitudes à proscrire. Dans un tel schème, l'enfant ne pouvait faire autrement que de manifester sans cesse une profonde inadéquation par rapport à ce qui était attendu de lui. En effet, comme la satisfaction de tout besoin physiologique était vue par le pasteur comme un signe de faiblesse, et donc comme une voie d'entrée pour Satan, et que l'expression des besoins affectifs et psychologiques était également vue comme une marque de faiblesse, l'enfant pouvait difficilement répondre aux attentes à son égard. Si cette inadéquation n'avait pas été interprétée comme une manifestation de l'emprise de Satan sur son âme, le parent aurait pu plus facilement adopter une attitude moins excessive devant ces manifestations somme toute normales, investissant alors une fonction parentale plus éducative que purement répressive, comme ce fut le cas à l'ÉBW. En ce sens, la dichotomisation exacerbée qui caractérisait les représentations de l'enfant a joué un rôle dans l'escalade des mauvais traitements infligés aux enfants. À la limite, pour certains parents, châtier physiquement son enfant équivalait non pas à le frapper lui ou elle, mais plutôt à libérer un être possédé du démon (Falwell, 1981; LaHaye, 1980). L'analogie avec l'exorcisme est certainement de mise surtout dans le cas de certains enfants identifiés comme particulièrement contaminés par le mal.

Par ailleurs, il importe de souligner que la représentation idéalisée de l'enfant propre au groupe devint, au fil de son évolution, un idéal partagé par les membres de l'ÉBW, et c'est en ce sens qu'il soutint désormais l'injonction à châtier systématiquement les enfants. Un tel idéal partagé exerça cependant un effet nuisible sur la capacité de jugement des membres puisqu'il en vint à prendre une importance démesurée pour eux. En effet, la poursuite d'un idéal groupal devint prioritaire notamment parce qu'il fut susceptible d'assurer la cohésion sociale du groupe et de renforcer le sentiment d'identité des membres, au détriment toutefois de leur jugement de la réalité (Casoni et Brunet, 2005). La fondation de la garderie et de l'école allèrent dans le sens de la poursuite de cet idéal, coûte que coûte, typique d'une organisation sociale associée à une philosophie dite de pureté (Casoni, 2000). Le fait d'avoir élargi le recours aux châtiments corporels aux nourrissons, 
d'avoir étendu son champ d'application à des comportements qui, de toute évidence, sont des manifestions d'immaturité, comme de pleurer de fatigue ou de maladie, ou sont associées à des différences individuelles de tempérament ou de rythme biologique, comme de ne pas dormir à l'heure prescrite, par exemple (Ellison,1996), et enfin d'avoir rendu certains châtiments publics donnent à penser que la poursuite de l'idéal de pureté, projeté sur le devenir des enfants, a certainement constitué un élément qui a pu participer à la situation de maltraitance en brouillant la capacité de jugement des membres de l'ÉBW (Casoni, 2000).

De telles réactions révèlent comment, à un certain point de l'évolution du groupe, les membres démontraient une grande incapacité à exercer leur jugement de façon individuelle. Ils ne semblaient plus réussir à prendre une distance par rapport au discours de leur pasteur et à se dégager des pressions du groupe pour s'interroger sur ce qui servirait véritablement le meilleur intérêt de leurs enfants. À cet égard, il faut toutefois remarquer que les représentations des enfants qui soustendirent les actions répressives à leur endroit furent très activement mises de l'avant par le pasteur, qui exerça incontestablement un rôle de leader auprès des fidèles. De fait, c'est lui qui insista sur une interprétation littérale particulière de certains versets bibliques choisis pour soutenir ces représentations de l'enfant. De plus, il prêcha régulièrement le besoin de châtier les enfants, obligea les membres à se plier à un protocole particulièrement sévère de punitions corporelles, exigea que tous les enfants fréquentent la garderie et l'école, y mit en place un régime très strict de châtiments corporels, donna lui-même fréquemment des punitions corporelles publiquement et élimina peu à peu toutes les possibilités pour les membres de se confronter à d'autres façons de voir en les isolant de plus en plus du monde extérieur. Ainsi, les injonctions de sa part pour imposer ces représentations négatives de l'enfant et contraindre moralement les parents à agir comme il l'exigeait - par ailleurs sous menace d'humiliation publique et d'expulsion en cas de refus - eurent beaucoup d'influence dans la dynamique groupale qui se développa pour soutenir le régime de punitions corporelles. L'effet combiné de tous ces éléments ne pouvait que mener à une impasse puisqu'il était impossible pour les enfants de répondre de façon satisfaisante aux attentes doctrinales et à l'idéal projeté sur eux. En outre, cette impasse pouvait difficilement donner lieu à autre chose qu'à une escalade dans la sévérité et la fréquence des punitions compte tenu des 
justifications doctrinales et des enjeux identitaires et idéologiques en cause.

La représentation idéalisée de l'enfant qui se dégage des données correspond en fait à celle décrite par Illick (1974) et Waltzer (1974) dans des contextes semblables, soit celle d'un enfant dont la subordination complète à l'autorité parentale donne l'impression qu'il est libéré des forces du mal. De plus, ces auteurs soulignent que la subordination aux parents est interprétée comme un signe du redressement et de la transformation de la nature de l'enfant, ce qui est non seulement rassurant pour les parents, mais constitue aussi un marqueur de leurs compétences parentales aux yeux de ceux qui partagent les mêmes représentations. La pression sociale interne pour faire partie du groupe des parents vus comme "compétents», puisque leurs enfants se rapprochaient le plus de la représentation idéalisée valorisée, a sans doute également constitué un élément important pour comprendre pourquoi les parents non seulement ne s'opposèrent pas aux châtiments corporels de leurs enfants - même lorsque ceux-ci devinrent de toute évidence excessifs - mais également pourquoi ils y participèrent activement.

Enfin, il importe d'ajouter que la mise sur pied de l'école et de la garderie a donné lieu à un nombre substantiel d'ayants droits d'administrer les châtiments corporels, ce qui en augmenta non seulement la fréquence potentielle, mais aussi la sévérité, car aucun lien affectueux avec l'enfant n'a pu y jouer un rôle d'atténuation. Le recours à la punition corporelle n'était contrebalancé ni par de l'affection envers les enfants, ni par l'influence de tiers, vecteurs des valeurs sociales, qui eurent pu faire office de contrepoids ou même de frein à la sévérité des membres qui n'avaient à répondre de leurs actes que devant le pasteur. Cet aspect joua également un rôle dans la situation du pasteur de l'ÉBW : aucun contrepoids à son orientation doctrinale ni aux règles mises en place pour châtier les enfants n'exista. Dans la première période de l'histoire de l'ÉBW, caractérisée par le partage de l'autorité en matière de doctrine, la tendance fondamentaliste du pasteur était freinée par la position doctrinale des évangélistes de longue date, nombreux et habitués à discuter la Bible, ainsi que par le droit de regard qu'avait la congrégation de Lennoxville sur celle de Windsor, ce qui constituait un contrepoids important puisqu'elle patronnait cette dernière. Cependant, le contrepoids représenté par l'Église de Lennoxville n'exerça plus sa fonction de tiers à partir du moment où le pasteur devint propriétaire des immeubles de l'Église. D'ailleurs, le pasteur a 
alors pu se permettre de défier les membres les plus anciens du groupe, soit ceux qui venaient de l'Église de Lennoxville. Puis, la scission eut lieu, laissant encore plus de latitude au pasteur. Le fait que l'ÉBW n'appartint à aucun regroupement, à aucune association ou hiérarchie religieuse pouvant exercer un rôle de surveillance par rapport aux interprétations doctrinales et aux pratiques qui s'y rattachaient ou encore exercer une fonction de tiers auprès duquel l'Église eut pu se confronter, a contribué à augmenter l'influence du pasteur sur les membres et sa marge de manœuvre quant aux orientations et aux pratiques.

En effet, aucun mécanisme d'arbitrage n'a existé, ni aucune instance supérieure n'a veillé à éviter les abus de pouvoir, d'interprétation ou de réglementation. Le pasteur fut donc libre d'interpréter la doctrine et d'imposer des normes et des sanctions sans être obligé de répondre de ses initiatives. C'est lors de la deuxième période de l'ÉBW que la centralisation du pouvoir autour de celui-ci accrut son autorité, d'abord sur les aspects doctrinaux, puis progressivement sur tous les aspects de la vie des membres, s'étendant à la sphère du travail puis progressivement, dès la troisième période, englobant la plupart des sphères de la vie quotidienne des membres. Cette emprise sur le vécu quotidien des fidèles rendit difficile toute remise en question de son autorité, car outre la sanction encourue sur le plan spirituel, les membres couraient aussi le risque de perdre leurs emplois ou leurs investissements dans le projet résidentiel de l'Église. Au cours de la quatrième période, par contre, l'emprise du pasteur s'affaiblit au fur et à mesure que des contrepoids apparurent. Ainsi, l'enquête conjointe de la police et de la DPJE l'obligea à décréter l'arrêt des punitions corporelles; de même, la peur de se voir retirer leurs enfants conduisit les membres du groupe à accepter de participer à l'enquête de la DPJE, puis à accepter des mesures qui allaient dans le sens opposé des pratiques prescrites par leur pasteur. Quant au rôle joué par l'enquête journalistique présentée par la Société Radio-Canada, il semble qu'elle ait eu un effet dans le départ de certains membres et ainsi contribué à la dissolution de l'Église. Bien qu'il semble avoir influencé les représentants des agences de contrôle social impliquées dans le dossier quant au moment choisi pour faire une descente dans le groupe, les données colligées ne permettent pas de se prononcer sur le rôle de ce reportage dans le choix du type d'intervention retenu. Par ailleurs, y attribuer un facteur causal dans la décision des agents de contrôle social d'intervenir à la suite de leurs enquêtes, 
qui étaient déjà fort avancées au moment de sa diffusion, serait spéculatif puisque les données recueillies ne permettent pas de le supposer. Néanmoins, il y a tout lieu de penser que ce reportage a pu contribuer à faire accepter l'intervention conjointe musclée, police/services de protection de la jeunesse, par l'opinion publique, vu la gravité des allégations de mauvais traitement qui y était formulées.

\section{Conclusion}

La maltraitance envers les enfants a été favorisée dès la fondation de l'Église baptiste de Windsor par son orientation conservatrice puisque le protestantisme conservateur véhicule un contenu doctrinal qui met de l'avant une représentation particulièrement négative de l'enfant tout en mettant l'accent sur le besoin de le redresser par la punition corporelle. Une autre composante de ce contenu doctrinal qui renforce l'injonction à utiliser les châtiments corporels est le besoin d'en faire les continuateurs de la foi de leurs parents. Cela conduit à un surinvestissement de l'enfant qui, puisqu'il entre en contradiction avec la représentation négative de celui-ci, accentue le besoin de le transformer. En ce sens, l'institutionnalisation de la punition corporelle a été favorisée, d'une part, en vertu de sa justification par l'univers symbolique partagé par le groupe et, d'autre part, parce que cette pratique visait à renforcer cet univers par sa perpétuation à travers la nouvelle génération (Berger et Luckmann, 1966).

Cela ne veut pas dire que tous les groupes qui partagent cet univers symbolique connaissent des dérives semblables à celle de l'ÉBW. Dans le cas de l'ÉBW, la centralisation du pouvoir entre les mains du pasteur a permis le glissement d'une interprétation conservatrice modérée de la Bible à une interprétation fondamentaliste; cela a mené à l'utilisation d'instruments pour administrer les punitions ainsi qu'à une codification stricte de leur utilisation qui a progressivement fait évoluer la punition corporelle vers la maltraitance. L'évolution dans le fonctionnement du groupe vers un fonctionnement sectaire a, à son tour, favorisé l'adoption d'une philosophie de la pureté qui a accentué le surinvestissement des enfants en tant que porteurs des idéaux du groupe, ce qui a contribué à l'augmentation de la fréquence et de la sévérité des châtiments en raison de l'incapacité des enfants à atteindre les idéaux projetés sur eux. Ce type de philosophie a aussi conduit à l'isolement de la congrégation 
de la société environnante, produisant une dynamique groupale dans laquelle le discours doctrinal concernant la punition corporelle n'a pu être confronté à aucun autre point de vue, ce qui contribua à miner l'exercice du jugement personnel des membres. Enfin, la place importante laissée au pasteur a joué un rôle déterminant dans la mise en œuvre, le maintien et l'aggravation de la situation de sévices physiques des enfants, et ce, d'autant plus qu'aucun contrepoids à ses injonctions n'existait.

L'articulation de ces différents éléments semble avoir donné naissance à un contexte microsocial où des actes graves de sévices physiques n'étaient malheureusement plus vus pour ce qu'ils étaient, soit des voies de fait. Ce ne sera qu'en réintroduisant une fonction tierce, exercée dans ce cas-ci par des agents de contrôle social - intervenants sociaux, policiers, juges -, que les membres de l'ÉBW purent, pour la plupart, prendre suffisamment de distance par rapport au discours de leur leader et à leur projet autarcique idéalisé, pour cesser de voir en leurs enfants ce qu'ils n'ont jamais été, soit des êtres dont l'âme appartenait à Satan. À cet égard, il peut être suggéré que l'intervention des agents de contrôle social, spécifiquement des intervenants sociaux auprès des membres de l'Église baptiste de Windsor a non seulement été salutaire, puisqu'elle a permis de mettre fin aux sévices dont étaient victimes les enfants du groupe, mais a aussi été optimale en termes de prévention, en ce qu'elle a empêché la réalisation du projet autarcique de l'Église, qui aurait isolé les membres et les enfants de façon telle qu'il serait devenu très difficile, voir impossible, d'intervenir pour les protéger. L'exemple de l'évolution des Apôtres de l'Amour Infini, installés dans un domaine retiré au Québec, peut être apporté comme illustration de ce vers quoi l'Église baptiste de Windsor aurait pu se diriger. Il devient en effet très difficile pour les agents de contrôle social d'intervenir pour protéger les enfants dont la sécurité ou le développement sont possiblement compromis dans un contexte de rupture avec la société, surtout lorsque cette rupture est associée à la poursuite d'un idéal de pureté. En ce sens, l'intervention de la DPJE peut être vue comme ayant été optimale en ce qu'elle a permis aux enfants de demeurer avec leurs parents tout en mettant fin à une dynamique groupale particulièrement nocive pour eux.

Des études de cas comme celle-ci contribuent sans conteste aux connaissances sur les groupes religieux minoritaires qui, de par leur nature même, en particulier par la difficulté qu'il y a d'y avoir accès, se prêtent difficilement à la recherche et mal à la recherche empirique 
quantitative. Cette étude a permis de mettre en lumière les interactions complexes, mais néanmoins déterminantes, entre style de leadership, univers symbolique et type de fonctionnement sectaire non seulement dans le recours aux punitions corporelles sur des enfants, mais surtout dans leur exacerbation à tel point qu'ils devinrent des sévices. Les constats isolés permettent ainsi d'identifier un type de processus qui ne peut que faire autrement que de mener à la dérive violente.

Les limites de cette étude doivent cependant être soulignées. D’abord, il y a lieu de rappeler celles inhérentes à toute reconstruction historique, qui sont désormais bien connues (Aron, 1938; Bloch, 1949). Bien que toutes les mesures requises aient été prises pour s'assurer de la validité des résultats présentés et de la justesse des analyses proposées, il va sans dire que cette étude, pas plus quaucune autre reconstruction historique d'ailleurs, ne peut s'enorgueillir d'être complètement neutre ni de faire totalement abstraction de son contexte d'émergence. Sans invalider les analyses présentées, cela en tempère toutefois le pouvoir explicatif puisque nul ne peut exclure l'influence d'un point aveugle chez les chercheurs dans leur travail de reconstruction. Voilà pourquoi il importe - plus que dans d'autres formes de comptes rendus de recherche - d'être exhaustif afin de donner au lecteur toutes les informations nécessaires pour critiquer le travail effectué.

D'intéressantes pistes de recherche se dessinent au terme de cette étude, notamment l'intérêt de faire une recherche auprès des membres de l'ÉBW afin de recueillir leur récit tant de leur vie au sein de l'Église, que de leur sortie et de leur retour à la vie en société plus large. Également, une recherche recueillant le point de vue des enfants d'alors, devenus depuis des adultes, permettrait de mieux comprendre leurs façons passées de s'expliquer les punitions corporelles dont ils ont été l'objet, ainsi que de cerner les effets qu'ils estiment que cette expérience a eu sur eux et sur leurs trajectoires de vie. Une précédente recherche suggère que les enfants qui font l'objet de châtiments corporels dans un contexte religieux ont tendance à s'identifier aux «forces du mal» qui sont explicitement vues comme agissant en eux (Depatie, 2005). À cet effet, il serait judicieux de vérifier si cela s'est présenté ainsi pour les enfants de l'ÉBW. Comme le régime de punitions corporelles dont ils ont fait l'objet est dorénavant connu, mieux en cerner les effets sur eux permettrait de baliser les interventions thérapeutiques souhaitables dans de telles circonstances. 


\section{Références}

Aron, R. (1938). Introduction à la philosophie de l'bistoire. Essai sur les limites de l'objectivité historique. Paris: Gallimard.

Bartskowski, J. P. (1995). Spare the Rod..., or spare the child - divergent perspective on conservative protestant child discipline. Review of Religion Researsh, 37 (2), 97-116.

Bartskowski, J. P., \& Ellison, C. G. (1995). Divergent Models of Chid-rearing in Popular Literature: Conservative Protestants vs the Mainstream Experts. Sociology of Religion, 56, 21-34.

Ben Barka, M. (1998). Les nouveaux rédempteurs: le fondamentalisme protestant aux États-Unis. Paris: Éditions de l'Atelier, Labor et Fides.

Berger, P., \& Luckmann, T. (1966) La construction sociale de la réalité. Paris: Armand Colin.

Bergeron, R. (1987). Les fondamentalistes et la Bible: quand la lettre se fait prison. Montréal: Fides.

Bloch, M. (1049). Apologie pour l'bistoire du métier d'bistorien. Paris: Armand Colin.

Bromley, D. G. (2002). Dramatic Denouements. In D. G. Bromley \& G. Melton (eds.), Cults, religion $\mathfrak{F}$ violence (11-41). Cambridge: Cambridge University Press.

Casoni, D. (2000). The Relation of Group Philosophy to Different Types of Dangerous Conduct in Cultic Groups. Cultic Studies Journal. 17, 143-167.

- (2001). Lorsque l'idéal gouverne. Étude des mécanismes psychologiques associés à un certain type de dérive sectaire. In J. Duhaime (ed.) La peur des sectes (83-95). Québec: Fides.

Casoni, D., \& Brunet, L. (2005). Processus groupal d'idéalisation et violence sectaire. Déviance et société, 29 (1), 75-88.

Chartrand, L. (1995). La Bible au pied de la lettre: le fondamentalisme questionné. Montréal: Médiaspaul.

Danso, H., Hunsberger, B., \& Pratt, M. (1997). The Role of Parental Religious Fundamentalism and Right-Wing Authoritarianism in Child-Rearing Goals and Practices. Journal for the Scientific Study of Religion, 36 (4), 496-511.

Depatie, C. (2005). Portrait de l'expérience et des opinions d'étudiants universitaires à l'égard de la punition corporelle. Mémoire de maîtrise. Université de Montréal, Montréal.

Dobson, J. (1976). The Strong-Willed Child: Birth Through Adolescence. Weathon, IL: Living Books/ Tyndale House.

- (1987). Parenting Isn't For Cowards. Dallas: Word Publishing.

Ellison, C. G. (1996). Conservative Protestantism and the Corporal Punishment of Childreen: Clarifying the Issues. Journal for the Scientific Study of Religion, 35 (1), 1-16.

Ellison, C. G., \& Sherkat, D. E. (1993) Conservative Protestantism and Support for Corporal Punishment. American Sociological Review, 58 (1), 131-144. 
Ellison, C. G., Bartkowski, J. P., \& Segal, M. L. (1996). Conservative Protestantism and the Parental Use of Corporal Punishment. Social Forces, 74 (3), 1003-1028.

El Mountacir, H. (1994). Les enfants des sectes. Mesnil-sur-l'Estrée (France): Fayard.

Erlanger, H. (1974). Social Class and Corporal Punishment in Childrearing: A Reassessment. American Sociological Review, 39, 68-85.

Falwell, J. (1981). Listen America. New York: Bantham.

Fath, S. (2004). Dieu bénisse l'Amérique. La religion de la Maison-Blanche. Paris: Éditions du Seuil.

Grasmick, H., Bursik, R. J., \& Kimpel, M. (1991). Protestant Fundamentalism and Attitudes Toward Corporal Punishment of Children. Violence and Victims, 6, 283-297.

Grasmick, H., Stout Morgan, C., \& Baldwin Kennedy, M. (1992). Support for Corporal Punishment in the Schools: A Comparison of the Effects of Socioeconomic Status and Religion. Social Science Quarterly, 73, 179-189.

Hervieu-Léger, D. (1988). Les fondamentalistes américains en politique; de l'évangélisme historique à la nouvelle droite chrétienne. Lumière et vie, 186, 19-30.

Illick, J. E. (1974). La crianza de los niños en Inglaterra y América del Norte en el siglo xvir. In DeMause, Ll., (ed.), Historia de la infancia (333-383). Madrid: Alianza editorial.

La Sainte Bible (Traduction de Louis Segond [1910], réédition de 1979). Genève: Société biblique de Genève.

LaHaye, B. (1977). How to Develop your child's temperament. Eugene, OR: Harvest House.

LaHaye, T. (1980). The Battle for the Mind. Old Tappan, New Jersey: Fleming H. Revell.

Nouveau Petit Robert (1994). Paris: Dictionnaires Le Robert.

Pacheco, A. (2002) Évolution des abus contre des mineurs dans l'Église baptiste de Windsor, Québec. Rapport de stage. Université de Montréal, Montréal.

Société Radio Canada (12 avril 1985). L'Église baptiste de Windsor. Le Point. Windsor: Société Radio Canada.

- (28 octobre1986). L'Église baptiste de Windsor, la suite. Le Point. Windsor: Société Radio Canada.

Waltzer J. F. (1974). Un periodo de ambivalencia: la infancia en América del Norte en el siglo xviII. In DeMause, Ll., (ed.) Historia de la infancia (384-418). Madrid: Alianza editorial.

Wilcox, W. B. (1998). Conservative Protestant Child-rearing: Authoritarian ou Autoritative? American Sociological Review, 63 (6), 796-809.

Willaime, J.-P. (1992). La précarité protestante. Sociologie du protestantisme contemporain. Genève: Éditions Labor et Fides.

Woodberry, R. D., \& Smith, C. S. (1998). Fondamentalism et al: Conservative Protestants in America. Annual review of Sociology, 24: 25-56. 


\section{Textes de loi}

Canada (1987). Code criminel annoté et lois connexes. Cowansville: Les Éditions Yvon Blais, Inc.

Québec (1979) Loi sur la protection de la jeunesse. LQR-P-34.1. Québec: Éditeur officiel du Québec.

Québec (1979). Loi sur les services de garde à l'enfance. LRQ-S-4.1. Québec: Éditeur officiel du Québec.

Recueils de jurisprudence du Québec (1986). Protection de la jeunesse - 224 (TJ). Québec: RJQ: 2711-2720.

ABSTRACT - Based on a case study, this article presents the evolution of a minority religious group of Quebec, the Baptist church of Windsor, towards a sectarian group whom, isolated from its social environment, resorted to severe child physical abuse. The children of the group were indeed subject to severe physical punishment that was considered child battering by the child protection agency and office of the attorney general. Through an exhaustive analysis of documents, complemented with testimonies of former members and interviews with key participants, the existence of the group is analyzed from origin to dissolution. Four stages in the evolution of the group were identified and its analysis highlights the elements contributing to the development of a sectarian and doctrinal context in which the use of severe physical punishment was generally accepted as legitimate.

RESUMEN - Este artículo tiene como objetivo estudiar, a partir de un caso único, la evolución de un grupo religioso minoritario de Quebec, la Iglesia bautista de Windsor, hacia un funcionamiento sectario y autoritario que lo aisló del ámbito social. Los niños de dicho grupo eran sometidos a severos castigos corporales que fueron considerados como maltrato físico por los servicios de protección de la infancia y por la oficina del procurador general. Gracias a un análisis documental exhaustivo acompañado de testimonios de ex miembros y de entrevistas con informantes clave ha sido posible reconstruir la historia de este grupo de su fundación a su disolución. Cuatro momentos en la evolución del grupo fueron identificados y su análisis permitió identificar los elementos que contribuyeron al surgimiento de un contexto grupal y doctrinal que legitimaba el recurso generalizado al maltrato físico de los menores del grupo. 